\title{
Belphégor
}

\section{Les Jeux olympiques de Berlin de 1936 dans la presse internationale}

Présentation générale

Paul Aron, Micheline Cambron, Gianni Haver, Marie-Ėve Thérenty and François Vallotton

\section{OpenEdition}

\section{Journals}

\section{Electronic version}

URL: http://journals.openedition.org/belphegor/824

DOI: 10.4000/belphegor.824

ISSN: 1499-7185

\section{Publisher}

LPCM

\section{Electronic reference}

Paul Aron, Micheline Cambron, Gianni Haver, Marie-Ėve Thérenty and François Vallotton, « Les Jeux olympiques de Berlin de 1936 dans la presse internationale », Belphégor [Online], 15-1 | 2017, Online since 06 July 2017, connection on 30 April 2019. URL : http://journals.openedition.org/belphegor/824 ; DOI : 10.4000/belphegor.824

This text was automatically generated on 30 April 2019.

\section{(c) $(1)$}

Belphégor est mis à disposition selon les termes de la Licence Creative Commons Attribution - Pas d'Utilisation Commerciale - Pas de Modification 4.0 International. 


\title{
Les Jeux olympiques de Berlin de 1936 dans la presse internationale
}

Présentation générale

\author{
Paul Aron, Micheline Cambron, Gianni Haver, Marie-Ève Thérenty and \\ François Vallotton
}

1 La présente livraison de la revue Belphégor publie les résultats d'une vaste étude internationale sur l'impact des Jeux olympiques de Berlin de 1936 dans la presse belge, française, québécoise et suisse.

2 L'événement, en lui-même, est bien connu et a fait l'objet de nombreuses publications. Les Jeux de 1936 ont été, de toute l'histoire de l'olympisme, ceux qui ont suscité le plus d'études. Pour la première fois en effet, l'histoire du sport affrontait l'épreuve de la politique à l'échelle mondiale et à grande ampleur. Les historiens ont parfaitement décrit l'importance que le régime nazi a accordée aux Jeux, la manière dont ils ont orchestré leur mise en scène spectaculaire. Ceux-ci marquaient à la fois le grand retour de l'Allemagne dans l'organisation du sport international, et une occasion unique de montrer au monde les réalisations du Reich. Par ailleurs, les débats occasionnés par les orientations racistes et militaires du régime ont suscité nombre de réactions. La manière dont le Comité international olympique (CIO) a géré ces questions est également bien connue.

Pourquoi donc y revenir, et sous l'angle particulier de l'étude de la presse écrite? Depuis une vingtaine d'années, la presse a pris une place de plus en plus importante dans la recherche. La numérisation des journaux, et notamment celle, rétroactive, de quotidiens anciens, a donné une nouvelle vie aux collections. Mais au-delà de cette opportunité, le constat qui fonde cet intérêt nouveau des chercheurs est avant tout lié à une nouvelle manière de penser la presse. Au lieu de considérer le journal comme une simple source historique, il convient de reconnaître que s'y jouent une série de phénomènes qui font du média non seulement le vecteur d'une information, mais aussi l'organisateur de celle-ci, selon des codes spécifiques progressivement mis au point pendant la période d'expansion de la presse écrite. Celle-ci s'est imposée comme la référence première de l'information au cours $d u \mathrm{XIX}^{\mathrm{e}}$ siècle, dépassant dans ce rôle la correspondance privée, la conversation 
ou les récits imprimés des siècles précédents. Pendant la même période, la presse a inventé à la fois un langage, des catégories d'expression (des rubriques), et mis en place tout un personnel aux fonctions différenciées (du pigiste au journaliste professionnel, du reporter à l'interviewer). La civilisation du journal décrit cela en détails ${ }^{1}$. Au début du $\mathrm{xx}^{\mathrm{e}}$ siècle, ce système s'est étendu à d'autres médias, comme la radio et le cinéma, sans toutefois en changer les données essentielles.

4 En même temps, avec l'internationalisation croissante des enjeux politiques, économiques et culturels, la presse a largement étendu son terrain d'investigation. Ses préoccupations, principalement nationales dans un premier temps, se sont ouvertes au monde entier. La colonisation, les conflits armés, les impérialismes ont nécessité l'envoi de reporters qui pouvaient rendre compte des événements lointains susceptibles d'influencer la vie quotidienne de tous, jusqu'aux provinces les plus reculées. Déjà bien présentes au xIX ${ }^{e}$ siècle, ces réalités n'ont fait que monter en puissance ensuite comme en témoigne la notion même, discutable mais significative, de "guerres mondiales". L'apparition de nouvelles techniques, comme la photographie de reportage, a rendu ces informations de plus en plus concrètes. De grandes agences internationales ont été créées pour faire circuler l'information qu'aucun journal, même le plus puissant, ne pouvait désormais maitriser avec ses seules ressources.

5 La question que les quatre équipes de recherche réunies dans le présent projet se sont posée est donc la suivante: comment la presse a-t-elle adapté ses pratiques à la mondialisation du xxe siècle ? Précisons : le mot clé ici est " pratiques ». Il ne s'agit pas de décrire l'appareil économique des médias, de raconter l'histoire des journaux, ni de retracer les transformations du métier, toutes études propres aux historiens de la presse. Les pratiques désignent des faits de langue et d'écriture, des manières de rendre compte des événements, l'articulation des discours en leur contexte. C'est pourquoi la dimension linguistique est ici essentielle. On privilégie la presse francophone, parce que l'on étudie des formes journalistiques qui héritent d'une histoire propre en formation depuis plus d'un siècle. Bien entendu, ces formes sont elles-mêmes influencées par d'autres traditions (notamment anglo-saxonnes) et dans certains cas, comme celui de Montréal, on ne peut se priver de la dimension comparative impliquée par la multiplicité des communautés rassemblées dans le même espace social.

6 À terme, le projet vise à confronter les expériences discursives de cette presse écrite face à des phénomènes d'ordre différent. Outre les Jeux, manifestation sportive et politique, on envisagera également le fait divers (en l'occurrence l'enlèvement du bébé Lindbergh et l'exécution de celui qui a été jugé comme son meurtrier), ainsi que des événements d'ordre culturel. Trois années de recherches communes devraient nous permettre de tirer les leçons générales de cette longue fréquentation des journaux.

7 Pour la presse écrite, la couverture d'un événement sportif international comme les Jeux comporte trois niveaux distincts. Il peut s'agir de rendre compte des résultats sportifs proprement dits, d'évoquer de manière sensible l'atmosphère des stades et de la ville de Berlin, les exploits des athlètes, et en particulier de la délégation nationale, ou enfin d'illustrer les manifestations sportives par le recours massif à la photographie. Outre le contact direct avec l'événement, par un correspondant spécial, les journaux bénéficient également des dépêches des agences de presse internationales et des services d'information ad hoc du Comité olympique allemand et du ministère de la propagande du Dr Goebbels. Car les Jeux de 1936 ne sont pas ceux d'Anvers de 1920 ou d'Amsterdam en 1928. 
8 Les Jeux de 1936 en effet ont été l'objet d'une attention toute particulière de la part du régime nazi en place depuis 1933 ; ils contrastent de ce point de vue, et volontairement, avec les Jeux de 1932, qui ont eu peu de participants. Pour la première fois, toutes les ressources d'un Etat ont été mobilisées en faveur d'un événement sportif, dont on attendait qu'il démontre la supériorité d'un régime, voire d'une race, sur le reste du monde. Rien n'a été négligé pour assurer la réussite de ce projet: infrastructures hôtelières et sportives, formation des athlètes et des équipes d'accueil, nettoyage des villes et gestion des foules. L'invention du parcours de la flamme olympique depuis la Grèce et de grandioses cérémonies d'inauguration et de clôture furent parmi les innovations les plus remarquables. Sur le plan médiatique, on a utilisé également toutes les ressources disponibles : cinéma, radio, presse écrite et même la télévision à ses tout débuts. On a accueilli et encadré les très nombreux journalistes étrangers accrédités par le CIO et le régime. Par l'importance même de ces investissements, et par la nature du régime qui les a accueillis, les Jeux de 1936 ont mis en question la neutralité sportive qui était (et est encore) le discours officiel du CIO.

9 La candidature du régime nazi à l'organisation des Jeux a suscité deux polémiques principales. L'une, menée par des associations juives et démocrates américaines, a plaidé pour le boycott des Jeux en réaction aux lois anti-juives nazies. Le mouvement a échoué parce que le CIO s'est rapidement déclaré satisfait par les garanties obtenues de la part des autorités allemandes, et parce que la ségrégation raciale américaine ne semblait pas $\mathrm{si}$ différente de celle pratiquée en Allemagne contre les non-Aryens. La seconde, plus politique, de la part des mouvements de gauche refusant l'orientation fasciste des Etats italien et allemand, a abouti à l'organisation d'Olympiades populaires prévues à Barcelone en juillet : les développements militaires de la guerre civile ont conduit à leur annulation.

10 Même rapidement esquissé, le contexte permet de comprendre les conditions particulières dans lesquelles s'est exercée la couverture journalistique des Jeux de 1936. Fallait-il ou non rendre compte de l'événement, et donc envoyer des reporters? Quelle serait la marge de manœuvre qui leur serait accordée par un régime militaire et dictatorial? Pouvait-on parler de sport sans évoquer la dimension symbolique des manifestations et des discours? Quel écho devait-on donner aux questions raciales et politiques? Ces questions, parmi bien d'autres, ont été au cœur du traitement médiatique des Jeux. Elles forment le cadre du déploiement rhétorique du travail journalistique.

11 La présente livraison comporte une contribution générale par pays concerné et une série d'études plus focalisées sur des points précis. Nous commencerons le dossier par les études sur la Suisse et la Belgique, parce qu'elles accordent une large place au contexte historique des Jeux et nous permettront d'éviter de répéter les principales données factuelles. Nous aborderons ensuite la France et le Québec, qui nous permettront de développer les aspects plus poétiques du dossier. Seront ensuite proposées des études focalisées sur la cérémonie d'ouverture, la photographie, le cas d'une athlète américaine interdite de participation (Eleanor Holm Jarrett), la journaliste Myrtle Cook et sur le caractère multilinguistique de la presse montréalaise. Un article méthodologique collectif conclut le dossier: il insiste sur la fécondité de ce genre d'étude pour la recherche actuelle et ses implications pédagogiques.

12 Pour les étudiants et les jeunes chercheurs de nos équipes, la découverte de la presse de 1936 ne laisse pas de surprendre. Par son importance quantitative d'abord, vu le nombre de périodiques publiés simultanément, reflétant tous les courants de l'opinion, les régions et les langues du moment. Cette abondance ne se reflète pas toujours dans les fonds 
disponibles : il a fallu procéder à des choix, selon la disponibilité des sources et l'état des collections. On verra cependant que la convergence des résultats de nos enquêtes suggère qu'une plus ample moisson n'aurait pas modifié nos conclusions principales.

$\mathrm{Au}$ départ de l'enquête, nous pensions que la dimension politique de l'événement, les compromis plus ou moins assumés avec le régime organisateur, concentreraient le gros de nos découvertes. Celles-ci ont été nombreuses, effectivement, et souvent plus contradictoires qu'on ne pouvait le penser. Les nuances apportées par la presse de droite, les mises en garde plus ou moins explicites sur la nature belliciste de l'Allemagne nazie, les réserves suggérées par une organisation et un enthousiasme trop bien encadrés pour être spontanés, ont stimulé l'imagination et la plume des envoyés spéciaux. Le contrôle des déplacements et des individus les a obligés à des euphémisations et à des contorsions qui s'apparentent parfois à une véritable rhétorique de la contrebande. D'où la rédaction de textes plus complexes et plus riches que de simples comptes-rendus sportifs. Mais la seconde surprise se situe ailleurs. Elle tient au véritable fossé qui sépare les images, les comparaisons, tout ce que l'on pourrait appeler l'impensé discursif des médias d'avantguerre de ceux d'aujourd'hui. Les journalistes de l'époque sont loin d'avoir intégré la réalité de la mondialisation et de l'égalité des sexes : ils s'étonnent de voir des athlètes japonais ou noirs américains battre leurs concurrents blancs et occidentaux; ils trouvent normal de multiplier les allusions désobligeantes aux femmes sportives, de déplorer les risques qu'elles font courir à leur féminité ou à leur «nature». Bien entendu, rares sont les femmes journalistes (Paule Hutzler pour Paris-Soir, Constance Coline pour Marianne et Myrtle Cook au Canada). Le racisme et le sexisme imprègnent la plupart des discours de presse, sans d'ailleurs pouvoir être assignés à des positions politiques particulières. En ce sens, la question de la race se révèle non réductible à ce qu'on nomme pudiquement à l'époque " la question juive ». La question de la hiérarchie des races, les pratiques ségrégationnistes ou discriminatoires se révèlent à la fois inscrites dans les discours, par le biais de stéréotypes, de clichés, de métaphores péjoratives ou mélioratives, et plus rarement, mais tout de même, discutées dans des textes critiques à l'endroit de Berlin ou à l'endroit des instances nationales mêlées à l'organisation des Jeux. Dans ces circonstances très particulières, le discours de presse se révèle une fois de plus comme un extraordinaire révélateur des référents sociaux. Il justifie par là-même qu'on se donne les moyens de le décrire et de le comprendre en profondeur.

\section{NOTES}

1. Kalifa Dominique, Régnier Philippe, Thérenty Marie-Ève \& Vaillant Alain, La Civilisation du journal. Histoire culturelle et littéraire de la presse au XIXe siècle, Paris, Nouveau Monde Éditions, «Opus Magnum », 2011. 
INDEX

Mots-clés: journal, presse, reportage, médias, jeux olympiques 1936, sport et nazisme

\section{AUTHORS}

\section{PAUL ARON}

Paul Aron est directeur de recherches au FNRS et professeur à l'ULB. Il est l'auteur de nombreux travaux en sociologie et en histoire de la littérature de langue française et de travaux sur les liens entre presse et littérature, notamment en Belgique francophone.

\section{MICHELINE CAMBRON}

Micheline Cambron est professeure au Département des littératures de langue française de l'Université de Montréal et membre du Centre de recherche interuniversitaire sur la littérature et la culture québécoise (CRILCQ). Elle consacre ses recherches et son enseignement à la littérature et à la culture québécoises des $\mathrm{XIX}^{\mathrm{e}}$ et $\mathrm{XX}^{\mathrm{e}}$ siècles. Elle a principalement travaillé sur les formes de l'utopie, les relations presse/littérature et les questions d'histoire littéraire et culturelle. Elle est également spécialiste des œuvres de Fernand Dumont et Paul Ricœur.

\section{GIANNI HAVER}

Gianni Haver est professeur associé de sociologie de l'image et d'histoire sociale des médias à l'Université de Lausanne. Il s'intéresse particulièrement aux productions médiatiques de la période de l'entre-deux-guerres et de la Deuxième Guerre mondiale et aux rapports entre histoire et médias. Il a notamment travaillé sur le cinéma et, plus récemment, sur les comics et la photo de presse. Il dirige la collection « Médias et histoire » aux éditions Antipodes.

\section{MARIE-ÈVE THÉRENTY}

Marie-Ève Thérenty est professeur de littérature française et directrice de l'équipe de recherche RIRRA21 de l'université Paul-Valéry Montpellier 3. Elle codirige avec Guillaume Pinson le site medias19.org. Elle est l'auteur de nombreux articles et livres sur les rapports entre littérature et médias, sur la poétique des supports et sur l'imaginaire médiatique des sociétés.

\section{FRANÇOIS VALLOTTON}

François Vallotton est professeur ordinaire d'histoire contemporaine à l'Université de Lausanne. Auteur de nombreuses contributions sur l'histoire culturelle et intellectuelle, il a consacré de nombreux ouvrages et articles à l'histoire du livre et de l'édition, à l'histoire de la presse ainsi qu'à l'histoire de la radio et de la télévision, dans une perspective suisse mais aussi transnationale. Il est également membre fondateur du Centre interdisciplinaire des Sciences historiques de la culture (Université de Lausanne). 